\title{
Inflammatory cytokine-induced expression of MASTL is involved in hepatocarcinogenesis by regulating cell cycle progression
}

\author{
LIYE CAO $^{1 *}$, WEN-JUAN LI ${ }^{2 *}$, JI-HONG YANG ${ }^{1}$, YU WANG $^{2}$, ZHI-JUAN HUA ${ }^{1}$, \\ DAN LIU ${ }^{3}$, YA-QING CHEN ${ }^{1}$, HAO-MIAO ZHANG $^{4}$, RUI ZHANG $^{1}$, JI-SEN ZHAO $^{1}$, \\ SHU-JIE CHENG ${ }^{1}$ and QUAN ZHANG ${ }^{1}$
}

\author{
${ }^{1}$ Department of Surgery, The Affiliated Hospital of Hebei University; ${ }^{2}$ College of Medicine, Hebei University, \\ Baoding, Hebei 071000; ${ }^{3}$ Department of Ultrasound Imaging, Zhuhai People's Hospital (Zhuhai Hospital \\ Affiliated with Jinan University), Zhuhai, Guangdong 519000; ${ }^{4}$ College of Medicine, \\ Tianjin Medical University, Tianjin 300070, P.R. China
}

Received April 27,2018; Accepted December 18, 2018

DOI: $10.3892 / \mathrm{ol} .2019 .9983$

\begin{abstract}
Microtubule associated serine/threonine kinase-like (MASTL) is the functional mammalian ortholog of Greatwall kinase (Gwl), which was originally discovered in Drosophila. Gwl is an essential kinase for accurate chromosome condensation and mitotic progression, and inhibits protein phosphatase $2 \mathrm{~A}$ (PP2A), which subsequently dephosphorylates the substrates of cyclin B1-cyclin-dependent kinase 1, leading to mitotic exit. Previous studies have indicated that MASTL has a critical function in the regulation of mitosis in HeLa and U2OS cell lines, though there is currently limited evidence for the involvement of MASTL in hepatocarcinogenesis. The results of the present study revealed that MASTL was inducible by the proinflammatory cytokines interleukin 6 (IL-6) and tumor necrosis factor alpha (TNF- $\alpha$ ), which promoted the proliferation and mitotic entry of human liver cancer cells. It was also determined that MASTL was significantly overexpressed in cancerous liver tissues compared with non-tumor liver tissues. Mechanistically, stimulation by IL- 6 and TNF- $\alpha$ induced the trimethylation of histone $\mathrm{H} 3$ lysine 4 (H3K4Me3) at the MASTL promoter to facilitate chromatin accessibility. Additionally, H3K4Me3 was associated with the activation of nuclear factor- $\mathrm{kB}$, which subsequently upregulated MASTL expression. These findings suggested that MASTL may have pivotal functions in the development of hepatocarcinoma, and that it may be a potential target for treatment.
\end{abstract}

Correspondence to: Professor Quan Zhang or Professor Shu-Jie Cheng, Department of Surgery, The Affiliated Hospital of Hebei University, 212 Yuhua East Road, Baoding, Hebei 071000, P.R. China E-mail: zzttmm0312@163.com

E-mail: chengshuj@126.com

${ }^{*}$ Contributed equally

Key words: microtubule associated serine/threonine kinase-like, inflammatory cytokines, hepatocarcinogenesis, mitosis

\section{Introduction}

The cell cycle involves a series of events controlled by a complex network of proteins, while mitosis is a cell division process of nuclear-envelope breakdown, chromosome condensation and spindle assembly (1). Cyclin-dependent kinase 1 (Cdk1)-cyclin B controls mitotic entry and exit. The inhibitory residues of Cdk1 are dephosphorylated by the dual-specificity phosphatase $\mathrm{Cdc} 25$, which is subsequently activated at the G2-M transition. This in turn phosphorylates multiple substrates by activating Cdk1-cyclin B (2,3). Previous studies have illustrated that protein phosphatase 2A (PP2A) negatively regulates the activation of Cdk1-cyclin B substrates, and promotes normal cell division (4). Greatwall (Gwl), a serine/threonine kinase originally identified in Drosophila (5) is essential for mitosis progression through PP2A inhibition by interacting with Alpha-endosulfine (Ensa)/cAMP-regulated phosphoprotein 19 (Arpp19) (6,7).

As a mammalian ortholog of Gwl, human microtubule associated serine/threonine kinase-like (MASTL) exhibits $50.2 \%$ sequence homology with Drosophila Gwl and $65.7 \%$ with Xenopus Gwl. Experiments in human cell lines, including HeLa or U2OS, have illustrated that MASTL has an essential function in regulating mitosis, similar to that of Gwl in Drosophila (8); there are also reports supporting the role of MASTL in lung cancer and thrombocytopenia $(9,10)$. Nevertheless, there is currently limited knowledge of the potential functions of MASTL in human diseases.

Liver cancer develops slowly from chronic liver diseases, including chronic hepatitis caused by hepatitis B virus (HBV) and hepatitis C virus (HCV) infection (11-13); as a result, genetic alterations and the loss of cell cycle regulation are two of the basic mechanisms of carcinogenesis (14-16). Chronic inflammation associated with viral infection favors the recruitment of macrophages that produce large quantities of proinflammatory cytokines (17). Studies have illustrated that TNF- $\alpha$ and IL- 6 are closely associated with the progression of liver cancer, and that the IкB kinase (IKK) and c-Jun NH2-terminal kinase (JNK) signaling pathways are involved in this process (18). However, the detailed mechanisms of hepatocarcinogenesis remain to be clarified. 
In the present study, MASTL was induced by TNF- $\alpha$ and IL- 6 in liver cancer cell lines, promoting cell proliferation by regulating the progression of the cell cycle. MASTL was markedly overexpressed in human liver cancer tissues compared with non-tumor liver tissues. TNF- $\alpha$ and IL- 6 promote the trimethylation of $\mathrm{H} 3 \mathrm{~K} 4$ to facilitate $\mathrm{NF}-\kappa \mathrm{B}$-induced $M A S T L$ transcription. Therefore, the data provided novel insights into the critical function of MASTL in the progression of live cancer.

\section{Materials and methods}

Liver cancer samples and sera. A total of 20 human liver cancer tissues and 20 adjacent non-tumor liver tissue specimens and their sera were used (Table I), we also recruited 20 healthy control subjects (Table II) from physical examination and the donating blood were collected for experiments. Patients with liver cancer were treated, and frozen tissue samples were obtained from The Affiliated Hospital of Hebei University, same as the healthy control subjects. The tumor type was confirmed by a pathologist. The study was approved by the Ethics Committee of The Affiliated Hospital of Hebei University, and written informed consent was obtained from all participants. All human materials were used in accordance with the Declaration of Helsinki Principles and relevant policies and regulations of China and the policies of the Institutional Review Board of the Hospital of Hebei University.

Cell culture. Human cell lines HepG2 and SUN387 were purchased from the Type Culture Collection of the Chinese Academy of Sciences (Beijing, China). The cell lines were maintained in RPMI-1640 medium (cat no. 11875; Gibco; Thermo Fisher Scientific, Inc., Waltham, MA, USA) and supplemented with $10 \%$ fetal bovine serum (cat no. 10437028; Gibco; Thermo Fisher Scientific, Inc.). Cells were incubated at $37^{\circ} \mathrm{C}$ in a humidified incubator, supplemented with $5 \% \mathrm{CO}_{2}$.

Reverse transcription-quantitative polymerase chain reaction (RT-qPCR). Total RNA was extracted from liver cancer cells using guanidinium thiocyanate (cat no. 5596026; Invitrogen; Thermo Fisher Scientific, Inc.). cDNA was generated by RT using oligo (dT) primers as outlined in the manufacturer's protocol (cat no. R233-01; Vazyme, Piscataway, NJ, USA). For the thermocyling conditions, $50^{\circ} \mathrm{C} 15 \mathrm{~min}, 85^{\circ} \mathrm{C} 5 \mathrm{sec}$. Target genes and controls were treated under the same conditions and analyzed by qPCR using SYBR Premix Ex Taq ${ }^{\mathrm{TM}}$ (cat. no. RR420L; Takara Biotechnology Co., Ltd., Dalian, China), according to the manufacturer's protocol. The primers used were as follows: MASTL forward, 5'-ATCCTGTATGCC ACATCAGAC-3', and reverse, 5'-TTTCTTCACCTTCTG GCCAAG-3'; GAPDH forward, 5'-GCACCGTCAAGGCTG AGAAC-3', and reverse, 5'-TGGTGAAGACGCCAGTGGA-3'. These results were normalized by the Pfaffi method (19) using CFX96 C1000 Touch Thermal Cycler (Bio-Rad Laboratories, Inc., Hercules, CA, USA).

Transfection and RNA interference (RNAi). RNAi experiments were performed using $30 \mathrm{nM}$ small interfering RNA (siRNA) oligos (Shanghai GenePharma Co., Ltd, Shanghai, China) by targeting human MASTL. Liver cancer cells were seeded at $2 \times 10^{5}$ cells per well in a 6 -well plate. Control siRNAs consisting of a scrambled sequence were used as a negative control. Transfection was performed with Lipofectamine ${ }^{\circledR}$ 2000 reagent (cat no. 11668019; Invitrogen; Thermo Fisher Scientific, Inc.), according to the manufacturer's protocol. The efficiency of RNAi was confirmed by western blot analysis $48 \mathrm{~h}$ post-transfection, and the phenotype was detected following confirmation of MASTL knockdown by siRNA. The MASTL siRNA sequence was 5'-GGACAAGTGTTATCGCTTA-3' (8).

Western blot analysis. Protein extracts were prepared with radioimmunoprecipitation assay buffer according to the manufacturer's protocol (cat no. 89900; Pierce; Thermo Fisher Scientific, Inc.). The protein concentration was detected by BCA assay (cat. no. CW0014S; CWBIO; Beijing, China). In brief, $40 \mu \mathrm{g}$ of whole cell lysate was separated by SDS-PAGE at $12 \%$ polyacrylamide and then transferred on to a PVDF membrane. Then, the membrane was blocked with 5\% BSA (cat. no. A8010; Beijing Solarbio Science \& Technology Co., Ltd., Beijing, China) in PBST for $1 \mathrm{~h}$ at room temperature and incubated with the primary antibodies overnight at $4^{\circ} \mathrm{C}$. Western blot analysis was performed using antibodies against MASTL (1:1,000) (cat no. ab86387; Abcam; Cambridge, MA, USA) and $\beta$-actin $(1: 5,000)$ (cat. no. 4967; Cell Signaling Technology, Inc., Danvers, MA, USA). The secondary antibodies conjugated with HRP $(1: 10,000)$ for $90 \mathrm{~min}$ at room temperature. The blots were developed using enhanced chemiluminescence reagent (cat. no. GERPN2109; GE Healthcare, Chicago, IL, USA) by GeneGnome XRQ (Syngene, Syngene Division of Synoptics Ltd; UK).

MTT assay. HepG2 and SUN3r7 cells were plated at $1 \times 10^{4}$ cells per well in 96-well plates and $10 \mu \mathrm{l}$ (cat no. C0009; Beyotime Institute of Biotechnology, Haimen, China) was added to the cells. MTT was removed $4 \mathrm{~h}$ post incubation, followed by pipetting of dimethyl sulfoxide to solubilize the formazan products. Absorbance was measured with a microplate reader (Gen5; BioTek Instruments, Inc., Winooski, VT, USA) at $570 \mathrm{~nm}$.

Flow cytometric analysis. HepG2 cells transfected with siRNA were harvested, washed with PBS, and fixed in $70 \%$ ethanol at $4^{\circ} \mathrm{C}$ overnight. The fixed cells were stained with propidium iodide (PI; cat no. C0080; Beijing Solarbio Science $\&$ Technology Co., Ltd.) at $37^{\circ} \mathrm{C}$ for $30 \mathrm{~min}$ prior to analysis via fluorescence-activated cell sorting (FACS) (Becton, Dickinson and Company, Franklin Lakes, NJ, USA). The cell cycle profiles were interpreted by BD CellQuest Pro software version 5.1 (BD Biosciences; Becton, Dickinson and Company).

Generation of vectors. Briefly, to create the MASTL kinase-dead mutation, the nucleotide residues 718-816 of $M A S T L$ were deleted. The DNA coding sequences of MASTL and the kinase-dead mutant of MASTL were cloned into the pcDNA3.1 eukaryotic expression vectors (Invitrogen; Thermo Fisher Scientific, Inc., USA) and were confirmed by sequencing.

Immunocytochemistry (IHC) and immunofluorescence histochemistry (IHF). Cells were cultured overnight on a 
Table I. Patient clinical characteristics.

\begin{tabular}{ccll}
\hline Patient no. & Age & Sex & Histology \\
\hline 1 & 80 & Female & Hepatocellular carcinoma \\
2 & 32 & Female & Hepatocellular carcinoma \\
3 & 59 & Female & Hepatocellular carcinoma \\
4 & 61 & Female & Hepatocellular carcinoma \\
5 & 59 & Female & Hepatocellular carcinoma \\
6 & 67 & Female & Hepatocellular carcinoma \\
7 & 54 & Female & Hepatocellular carcinoma \\
8 & 56 & Male & Hepatocellular carcinoma \\
9 & 77 & Female & Hepatocellular carcinoma \\
10 & 60 & Female & Hepatocellular carcinoma \\
11 & 35 & Female & Hepatocellular carcinoma \\
12 & 57 & Female & Hepatocellular carcinoma \\
13 & 30 & Female & Hepatocellular carcinoma \\
14 & 67 & Female & Hepatocellular carcinoma \\
15 & 67 & Female & Hepatocellular carcinoma \\
16 & 60 & Male & Hepatocellular carcinoma \\
17 & 64 & Male & Hepatocellular carcinoma \\
18 & 43 & Male & Hepatocellular carcinoma \\
19 & 42 & Male & Hepatocellular carcinoma \\
20 & 81 & Male & Hepatocellular carcinoma \\
\hline & & &
\end{tabular}

Table II. The characteristics of healthy subjects.

\begin{tabular}{lll}
\hline No. & Age & Sex \\
\hline 1 & 35 & Female \\
2 & 52 & Female \\
3 & 40 & Female \\
4 & 76 & Male \\
5 & 65 & Male \\
6 & 37 & Male \\
7 & 37 & Female \\
8 & 30 & Male \\
9 & 58 & Male \\
10 & 52 & Male \\
11 & 50 & Female \\
12 & 38 & Female \\
13 & 59 & Male \\
14 & 75 & Male \\
15 & 35 & Male \\
16 & 39 & Male \\
17 & 68 & Male \\
18 & 38 & Male \\
19 & 40 & Female \\
20 & 44 & Female \\
\hline & & \\
\hline
\end{tabular}

glass coverslip and fixed using $4 \%$ paraformaldehyde for $30 \mathrm{~min}$ at room temperature. The cells were incubated with primary anti-MASTL antibody and secondary fluorescein isothiocyanate (FITC)-goat anti-rabbit IgG antibody (cat no. ab6717; Abcam; USA). DAPI (cat. no. C1002; Beyotime Institute of Biotechnology) was employed for nuclear staining. Paraffin sections were compared with non-tumor liver samples fixed using 4\% paraformaldehyde overnight at room temperature (tissue sections $8 \mathrm{~mm}$ ), $0.5 \%$ Triton $\mathrm{X}-100$ in PBS was used for reducing non-specific interactions at room temperature for $1 \mathrm{~h}$, and subsequently stained with anti-MASTL antibody (1:100) and FITC-goat anti-rabbit IgG antibodies (1:50). Images were acquired using a fluorescence microscope (magnification, x100) (E600; Nikon Corporation, Tokyo, Japan) and the results were evaluated by fluorescence signal intensity using Zeiss 510 META software version 3.5.

Enzyme-linked immunosorbent assay (ELISA). IL-6 and TNF- $\alpha$ quantification in healthy and liver cancer patient sera by ELISA (cat. no. EK106/2\&EK1822; MULTI SCIENCES, Beijing; China) according to the manufacturer's protocol. The concentrations of IL- 6 and TNF- $\alpha$ were calculated based on standard curves provided with the kits, and the results of IL-6 and TNF- $\alpha$ were expressed in $\mathrm{pg} / \mathrm{ml}$.

Chromatin immunoprecipitation (ChIP) assay. Cells were processed according to the protocol described in the ChIP Assay kit (cat no. P2078; Beyotime Institute of Biotechnology, Haimen, China) with anti-H3K4Me3 antibody (1:100) (cat no. 07-473; EMD Millipore, Billerica, MA, USA). Magnetic protein $\mathrm{G}$ beads (cat no. 88847; Pierce; Thermo Fisher Scientific, Inc.) were used to pull-down the antibody-chromatin complexes. The ChIP primers used were as follows: MASTL pro (0-0.2 K) forward, 5'-TTAAGACTTTCTACA GCTT-3', and reverse, 5'-TGAGCAGGAAGCGAGTCC-3'. The NF- $\kappa$ B inhibitor pyrrolidine dithiocarbamate (PDTC; cat. no. P8765) was purchased from Sigma-Aldrich (Merck KGaA, Darmstadt, Germany). The STAT3 inhibitor NSC74859 (cat. no. SD4794) was purchased from Beyotime (Beyotime Institute of Biotechnology).

Statistical analysis. One-way ANOVA followed by Newman-Keuls post-test was used for multi-group comparisons. The two-tailed Student's t-test of SPSS 18.0 (SPSS, Inc., Chicago, IL, USA) was used for two-group comparisons. All the experiments were repeated at least three times. Results are displayed as the mean \pm standard error of the mean. $\mathrm{P}<0.05$ was considered to indicate a statistically significant difference.

\section{Results}

Inducible expression of MASTL by inflammatory cytokines. Cancer of the liver is one of the most common causes of cancer-associated mortality worldwide (11); in China, the majority of liver cancer cases occur due to sustained, chronic infection with HBV or HCV $(12,13,20)$. Numerous studies have confirmed that inflammatory cytokines, including IL- 6 and TNF- $\alpha$, secreted by Kupffer cells and hepatocytes, contribute to the development of hepatocacinogenesis (21-23). Additionally, these inflammatory cytokines may activate signal transducer and activator of transcription 3 (STAT3) and $\mathrm{NF}-\kappa \mathrm{B}$ to induce hepatocarcinogenesis $(24,25)$, though the detailed mechanisms are yet to be clarified. 
To investigate whether IL- 6 or TNF- $\alpha$ may affect the expression of MASTL in the human liver cancer cell lines HepG2 and SUN387, these cells were stimulated with 100 $\mathrm{pg} / \mathrm{ml}$ TNF- $\alpha$ or IL- 6 for $1-5$ days. RT-qPCR and western blotting were performed to determine the mRNA and protein expression levels of MASTL. MASTL mRNA expression was induced in HepG2 and SUN387 cells by stimulation with IL-6 or TNF- $\alpha$ (Fig. 1A and B). Similarly, MASTL protein expression was increased by the introduction of IL- 6 and TNF- $\alpha$ (Fig. 1C and D).

Knockdown of MASTL leads to mitotic arrest in liver cancer cell lines. A number of studies have demonstrated that $\mathrm{Gwl}$ kinases serve a critical role in regulating mitosis in Drosophila and Xenopus. In addition, Gwl kinases inhibit the activation of PP2A by interacting with Ensa/Arpp19 $(26,27)$. Previous studies of MASTL confirmed that it had a similar function in regulating mitotic entry and cytokinesis in human cell lines, including HeLa and U2OS (8).

To demonstrate the function of MASTL in HepG2 cells, MASTL was knocked down with siRNA (Fig. 2A). FACS analysis was performed following PI staining to examine whether MASTL silencing altered cell cycle progression. The results indicated that cells transfected with siRNA-MASTL were arrested at the G2/M phase compared with the control cells (Fig. 2B and C). Morphological differences were observed by immunofluorescence. Specifically, HepG2 cells treated with siRNA exhibited a greater proportion of multinuclear cells compared with control cells (Fig. 2D and E). This indicated that the cells failed to enter mitosis and complete cell division. These results were consistent with previous reports $(8,28)$, which demenstrated that cells depleted of MASTL by siRNA remain in the G2 phase, and exhibit slow chromosomal condensation.

Based on the FACS data, it was hypothesized that knockdown of MASTL may inhibit liver cancer cell proliferation. To confirm this hypothesis, an MTT assay was performed to examine the proliferation of HepG2 and SUN387 cells transfected with MASTL siRNA or control siRNA, and western blotting was performed to test the efficiency of silencing in SUN387 cells (Fig. 2A, right). The results indicated that the proliferation of MASTL-silenced HepG2 and SUN387 cells was significantly inhibited compared with the control samples (Fig. 2F), suggesting that MASTL influences the proliferation of liver cancer cells.

Overexpression of MASTL promotes cell proliferation and cell cycle progression. The function of MASTL in cell cycle progression and cell proliferation was examined by overexpression in HepG2 and SUN387 cell lines. Exogenous expression of MASTL was confirmed using an anti-Flag antibody (Fig. 3A), and a subsequent MTT assay revealed that overexpression of MASTL in HepG2 and SUN387 cells resulted in more rapid proliferation, compared with the empty vector controls (Fig. 3B). These results are partially consistent with the MASTL silencing data in these cell lines (predominantly in HepG2), further confirming the role of MASTL in liver cancer proliferation.

Furthermore, MASTL wild type and kinase-dead vectors were transfected into HepG2 cells to assess alterations in
Table III. Expression of MASTL protein in liver cancer and non-tumor liver tissues by immunofluorescence histochemistry.

\begin{tabular}{lcrc}
\hline & \multicolumn{2}{c}{ MASTL expression, $\mathrm{n}$} & \\
\cline { 2 - 3 } Histology & High (\%) & Low (\%) & Total cases, $\mathrm{n}$ \\
\hline Tumor & $14(70)$ & $6(30)$ & 20 \\
Non-tumor & $4(20)$ & $16(80)$ & 20 \\
\hline
\end{tabular}

MASTL, microtubule associated serine/threonine kinase-like.

morphology (Fig. 3C). The nuclei of HepG2 cells transfected with the wild type MASTL vector were much smaller, and aggregated together compared with those transfected with kinase-dead vector, indicating that MASTL promotes liver cancer cell proliferation. FACS analysis was performed with cells expressing mutant MASTL and the corresponding empty vector. The results indicated that the number of cells expressing mutant MASTL in the G1 phase was significantly decreased compared with the control (Fig. 3D and E). Taken together, this suggested the involvement of MASTL in liver cancer cell proliferation by regulating cell cycle progression. These results were consistent with previous reports $(8,28)$ which illustrated that MASTL knockdown impaired cell proliferation.

Increased expression of MASTL in liver cancer. To confirm whether the inflammatory cytokines IL- 6 and TNF- $\alpha$ induced MASTL expression in liver cancer tissues, 20 patients with liver cancer and 20 healthy control subjects were recruited. The results indicated higher concentrations of IL- 6 and TNF- $\alpha$ in the sera of diseased patients compared with those of the control subjects (Fig. 4A). IHF and IHC results revealed that 14 of the 20 diseased patients $(70 \%)$ exhibited high expression of the MASTL protein, and all of these patients were chronically infected with HBV. Chronic HBV infection was determined by persistent HBV surface antigenemia lasting more than six months. By contrast, 4 of the 20 matched non-tumor liver tissues (20\%) displayed increased MASTL protein expression (Fig. 4B). Lower expression of the MASTL protein was revealed in 16 of the 20 non-tumor liver tissues (Table III). These data indicated that the high expression of MASTL promoted liver cancer carcinogenesis.

IL-6 and TNF- $\alpha$ promote methylation of $\mathrm{H} 3 \mathrm{~K} 4$ to facilitate $N F-\kappa B$-mediated MASTL transcription. The mechanisms involved in the IL- 6 and TNF- $\alpha$-induced MASTL expression in liver cancer cell lines were investigated. A ChIP assay was performed to analyze whether stimulation with cytokines effected the methylation of histone H3K4 at the MASTL promoter, which critically influences the regulation of transcription by increasing chromatin accessibility. The results revealed that by day $4, \mathrm{H} 3 \mathrm{~K} 4 \mathrm{me} 3$ at the MASTL promoter was significantly increased in HepG2 cells treated with IL-6 or TNF- $\alpha$ (Fig. 4C). This suggested that cytokine stimulation of liver cancer cells promoted chromatin accessibility at the MASTL promoter, inducing MASTL expression. 
A

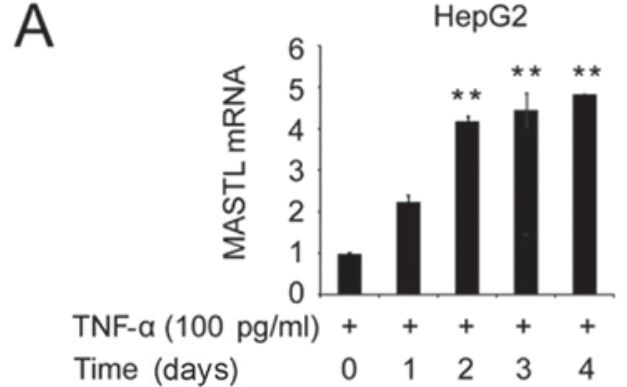

B
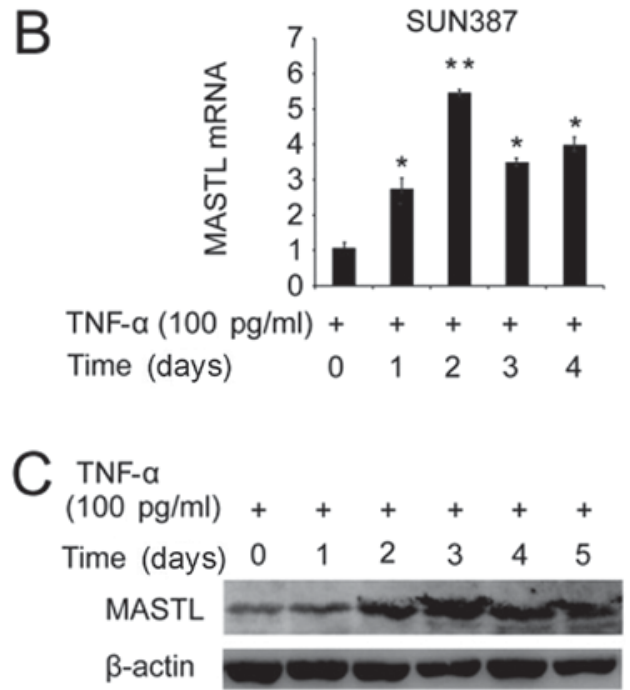

IL6

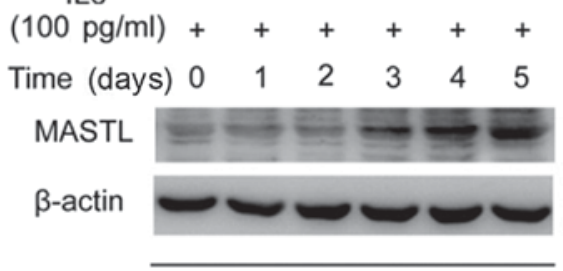

HepG2
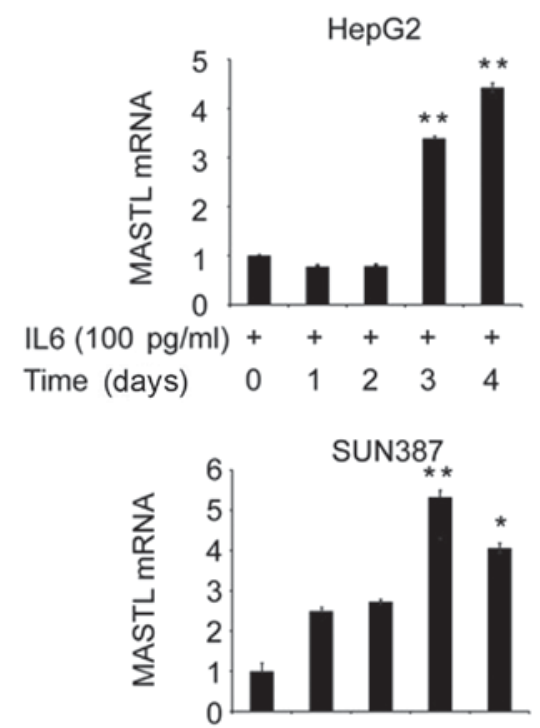

$\operatorname{IL} 6(100 \mathrm{pg} / \mathrm{ml})+++++$

Time (days) $\quad 0 \quad 1 \quad 2 \quad 3 \quad 4$

Figure 1. Induction of MASTL expression in HepG2 and SUN387 cells. Cells were stimulated with (A) TNF $\alpha$ and (B) IL-6. Examination of relative MASTL mRNA expression in HepG2 cells treated with TNF- $\alpha$ or IL-6 $(100 \mathrm{pg} / \mathrm{ml})$ at the indicated time points by reverse transcription-quantitative polymerase chain reaction. The expression of MASTL in HepG2 and SUN387 cells were measured (C and D), and $\beta$-actin was used as an internal control. Data are representative of three independent experiments. Results are displayed as the mean \pm standard error of the mean. ${ }^{*} \mathrm{P}<0.05,{ }^{* *} \mathrm{P}<0.01$ vs. respective control. MASTL, microtubule associated serine/threonine kinase-like; TNF- $\alpha$, tumor necrosis factor $\alpha$; IL-6, interleukin 6.

Numerous studies have demonstrated that IL- 6 and TNF- $\alpha$ signaling promotes the proliferation of liver cancer cells by NF- $\mathrm{KB}$ activation (29-32); therefore, the role of NF- $\mathrm{\kappa B}$ in IL-6 and TNF- $\alpha$-induced expression of MASTL was investigated. HepG2 cells were pretreated with the NF- $\mathrm{BB}$ inhibitor PDTC, and the MASTL mRNA expression level was measured in response to IL-6 or TNF- $\alpha$ stimulation. The promotion of MASTL mRNA expression was inhibited by PDTC (Fig. 4D). In addition, a ChIP assay was performed, which revealed that on day 4, H3K4me3 at the MASTL promoter was significantly decreased in HepG2 cells treated with PDTC (Fig. 4E). Thus NF- $\kappa$ B activation was required to induce MASTL mRNA expression by IL-6 or TNF- $\alpha$.

In summary, stimulation of liver cancer cells with IL-6 or TNF- $\alpha$ promoted trimethylation of histone $\mathrm{H} 3$ lysine 4 at the MASTL promoter to facilitate chromatin accessibility, and $\mathrm{NF}-\kappa \mathrm{B}$ was involved in cytokine-induced MASTL mRNA expression.

\section{Discussion}

Chronic hepatitis as a result of $\mathrm{HBV}$ or $\mathrm{HCV}$ infection is associated with cancer of the liver. The transformation and development of cancer occurs in response to numerous pathological events, including cell damage, oxidative stress, compensatory regeneration and proliferation (12). Various immune cells, including lymphocytes, macrophages, natural killer cells, natural killer T cells and dendritic cells are involved in chronic hepatitis-associated liver cancer (27). TNF- $\alpha$ and IL-6 are secreted by immune cells or hepatocytes, leading to either promotion or inhibition of multiple hepatocarcinogenesis-associated genes (33). In addition, cell cycle dysregulation may trigger carcinogenesis, and previous studies have illustrated that MASTL, the mammalian ortholog of Gwl, has an essential role in the entry to and exit from mitosis in human cell lines, including HeLa and U2OS (8). In the present study, the effects 
A

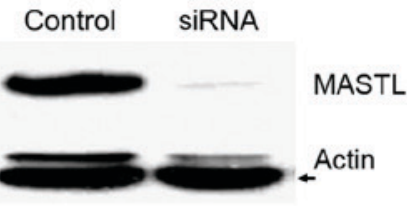

HepG2

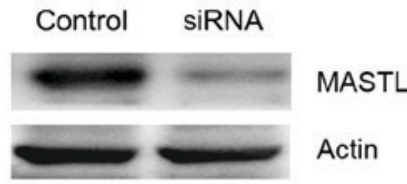

SUN387

B

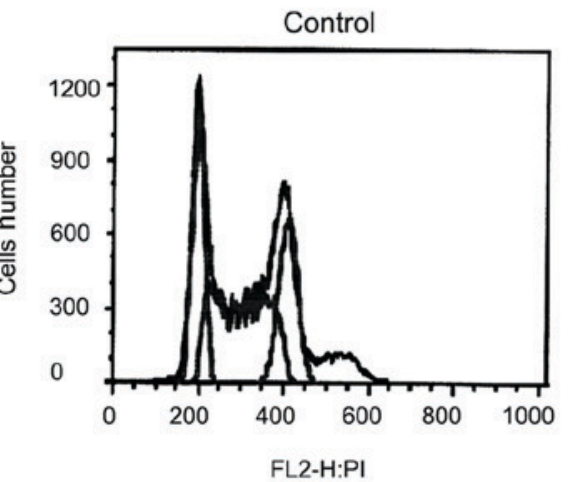

Watson pragmatic:

$\% \mathrm{G} 1=21.5 ; \% \mathrm{~S}=33.7 ; \% \mathrm{G} 2=23.1$

Event count: 78288
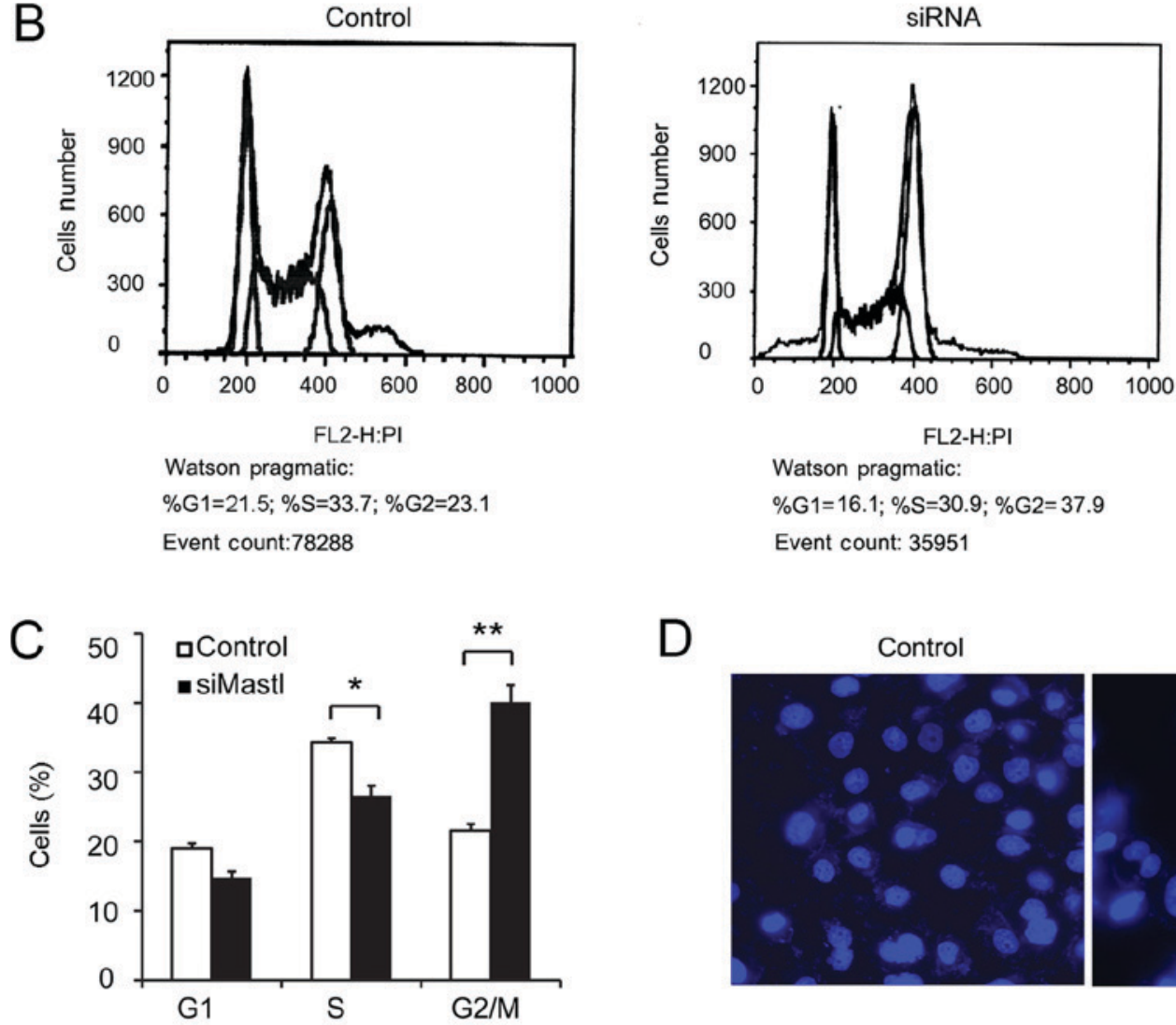

Watson pragmatic:

$\% \mathrm{G} 1=16.1 ; \% \mathrm{~S}=30.9 ; \% \mathrm{G} 2=37.9$

Event count: 35951

D
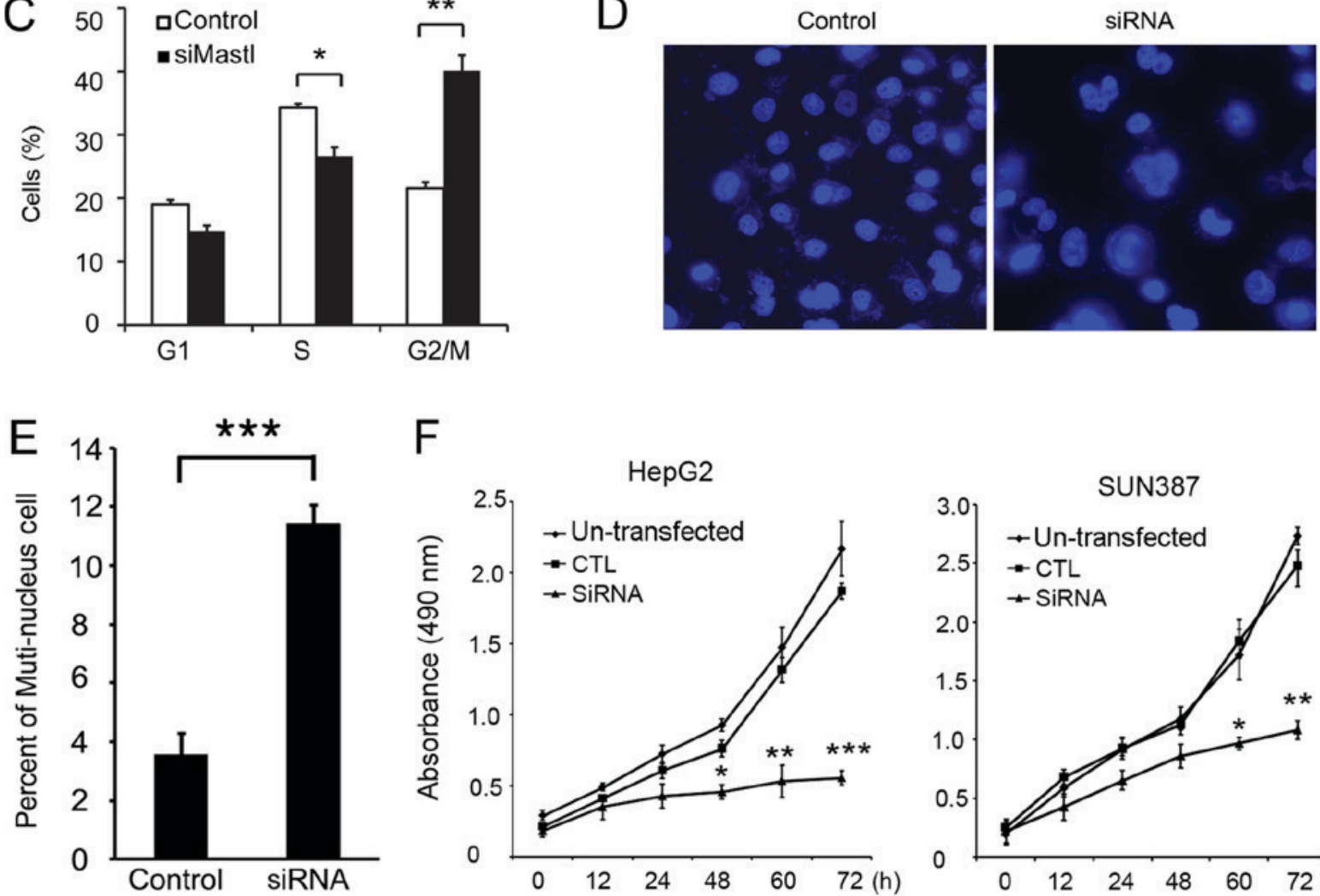

F
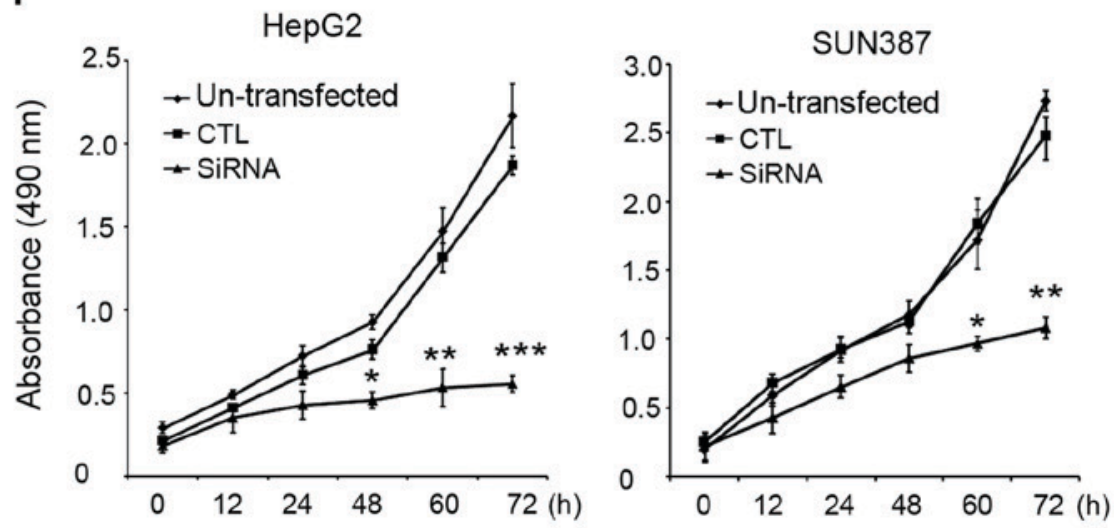

Figure 2. Effects on morphology and cell cycle progression of silencing of MASTL in liver cancer cell lines. (A) Knockdown of MASTL in HepG2 and SUN387 cells was confirmed by western blot analysis. (B) Fluorescence-activated cell sorting analysis and (C) quantification confirmed that the silencing of MASTL in HepG2 cells facilitated G2/M phase arrest (37.9\%) compared with the control (23.1\%). (D) DAPI staining (magnification, x100) and (E) quantification revealed increased multinuclear cells in HepG2 with silenced MASTL compared with the control. Cells transfected with scrambled siRNA served as a control. (F) An MTT assay was used to determine the relative cellular proliferation of SUN387 and HepG2 cells transfected with siRNA-MASTL, measured at $490 \mathrm{~nm}$. Experiments were repeated three times. Results are displayed as the mean \pm standard error of the mean. ${ }^{*} \mathrm{P}<0.05,{ }^{* * *} \mathrm{P}<0.01,{ }^{* * *} \mathrm{P}<0.001$ vs. respective control. MASTL, microtubule-associated serine/threonine kinase-like; siRNA, small interfering RNA; CTL, control; PI, propidium iodide.

of the proinflammatory cytokines TNF- $\alpha$ and IL- 6 on MASTL expression were investigated in liver cancer cell lines. The data revealed that mRNA and protein expression were induced in response to TNF- $\alpha$ or IL-6 stimulation, suggesting that MASTL serves a prominent role in chronic hepatitis-associated liver cancer. Moreover, the NF-kB inhibitor PDTC, and the STAT3 
A

Vector MASTL Vector MASTL

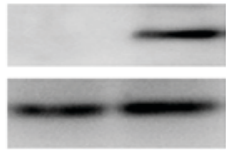

HepG2

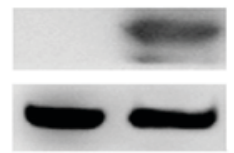

SUN387
Flag-MASTL

$\beta$-Actin
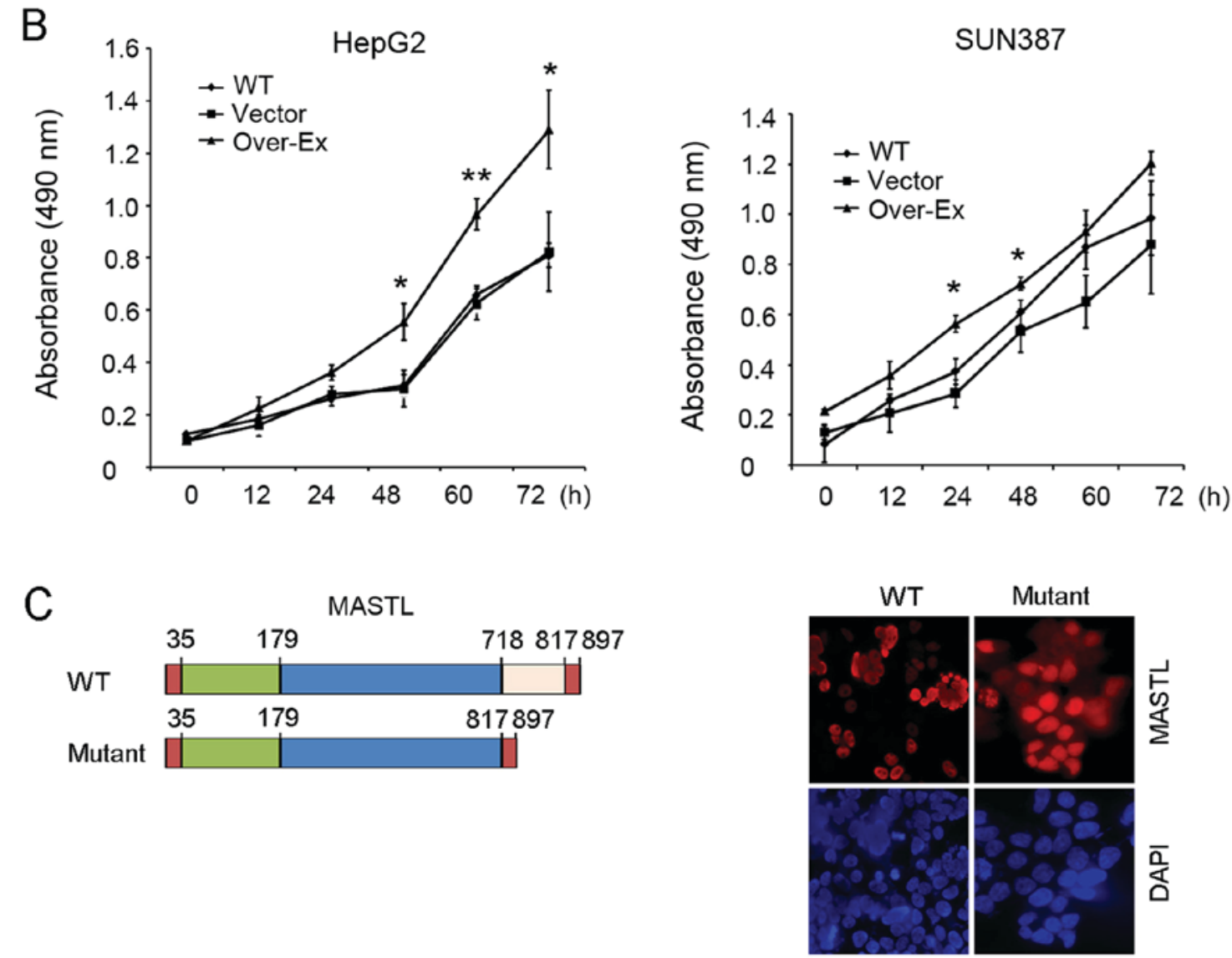

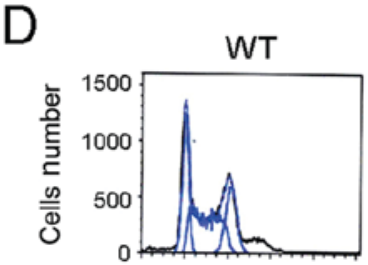

FL2-H:PI

Watson pragmatic: $\% \mathrm{G} 1=28.3 ; \% \mathrm{~S}=38$; $\% \mathrm{G} 2=22.7$

Event count:78288

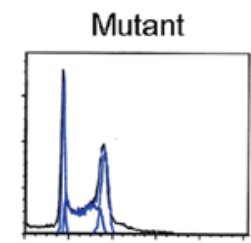

FL2-H:PI

Watson pragmatic: $\% \mathrm{G} 1=18.2 ; \% \mathrm{~S}=33.7$; $\% G 2=23.1$

Event count:78288
$E$

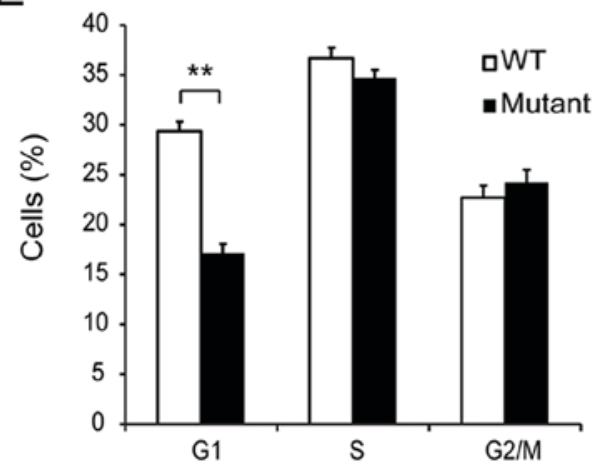

Figure 3. Effects of MASTL overexpression on the proliferation of liver cancer cell lines HepG2 and SUN387. (A) Overexpression of MASTL in HepG2 and SUN387 cells was confirmed by western blot analysis. Transfection of an empty vector was used as a control, and $\beta$-actin was used as a loading control. (B) An MTT assay was performed following overexpression of MASTL at the indicated time points. (C) Staining of WT or kinase-dead mutant MASTL expression with anti-flag in HepG2 cells by immunofluorescence (magnification, x100). (D) Cell cycle progression of HepG2 cells in overexpressing WT or mutant MASTL was examined using fluorescence-activated cell sorting. (E) The percentages of cells in the respective cell cycle phases. Experiments were repeated three times. Results are displayed as the mean \pm standard error of the mean. ${ }^{*} \mathrm{P}<0.05,{ }^{* *} \mathrm{P}<0.01$ vs. respective controls. MASTL, microtubule associated serine/threonine kinase-like; WT, wild-type. 

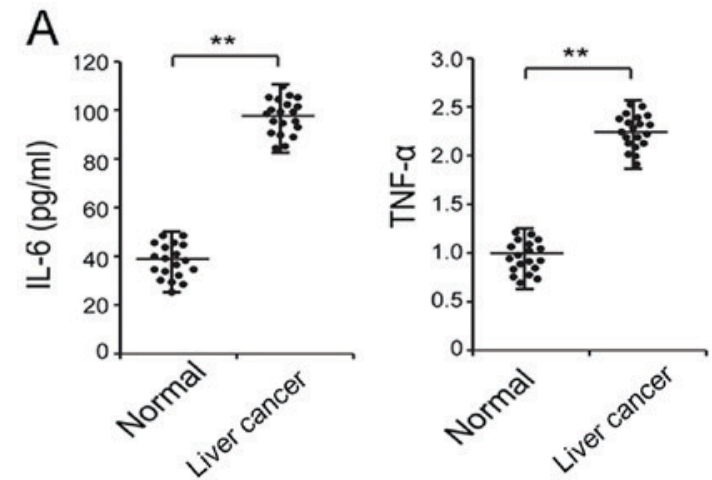

C

H3K4M3

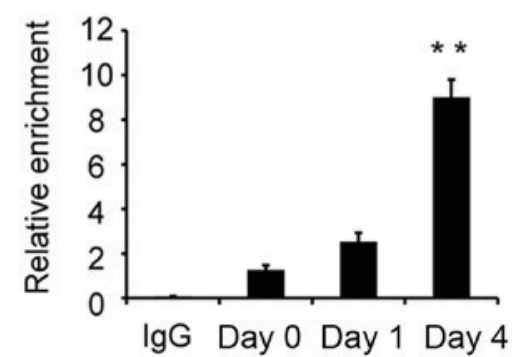

$\mathrm{D}$

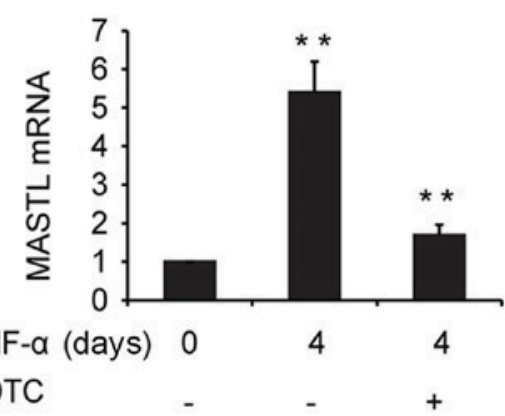

$\mathrm{E}$

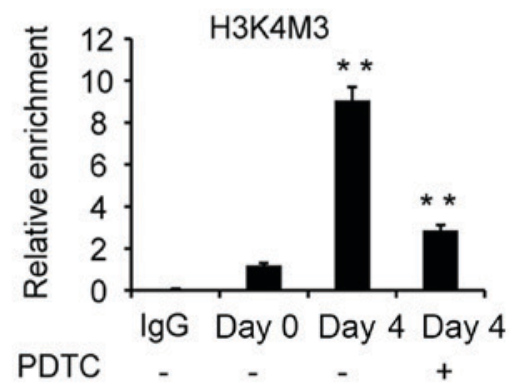

B
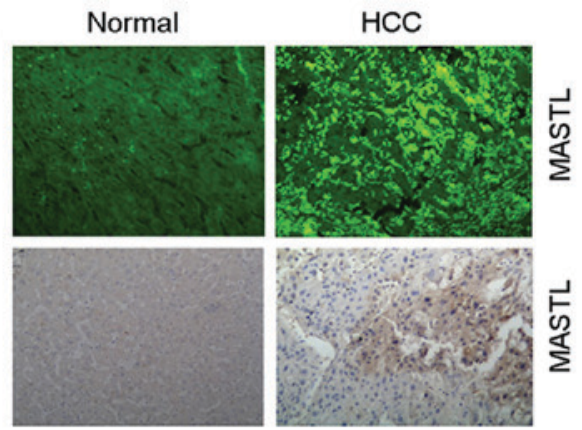

H3K4M3
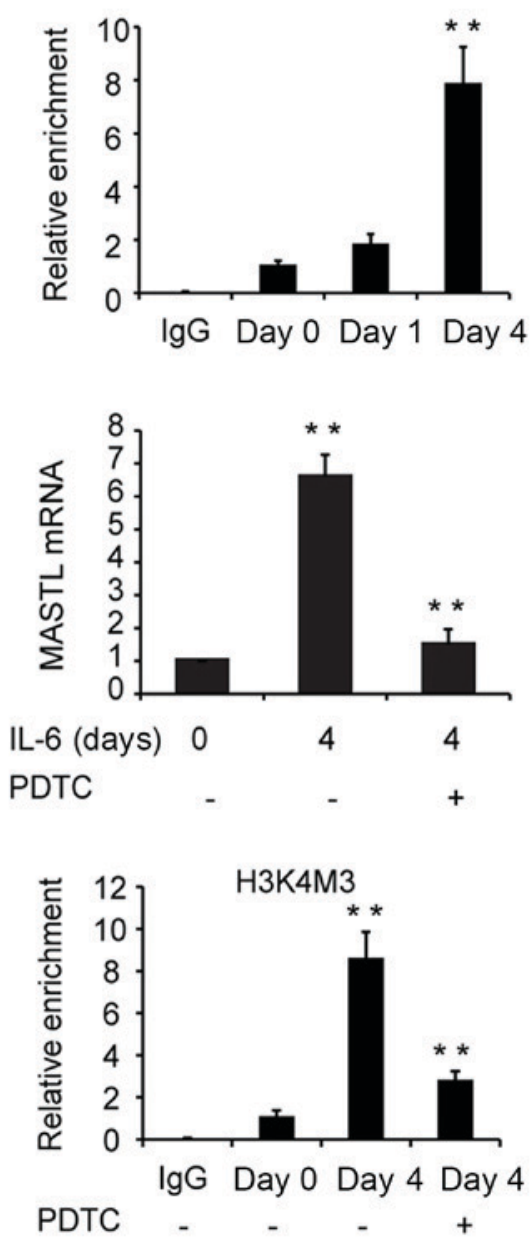

Figure 4. Expression of MASTL in human liver samples and the mechanisms involved in the IL-6 and TNF- $\alpha$-induced expression of $M A S T L$ in liver cancer cell lines. (A) IL-6 and TNF- $\alpha$ quantification in healthy and liver cancer patient sera by ELISA. (B) Detection of MASTL in liver cancer and non-tumor liver tissues by immunofluorescence or immunohistochemistry (magnification, x50). (C) ChIP assay of H3K4Me3 modification at the MASTL promoter in HepG2 cells treated with IL-6 (right) and TNF- $\alpha$ (left) at indicated time points. (D) MASTL mRNA expression in HepG2 cells pretreated with or without PDTC (30 $\mu$ mol/l) in response to IL-6 and TNF- $\alpha$ stimulation. (E) ChIP assay of H3K4Me3 modification pretreated with or without PDTC at indicated time points. Experiments were repeated three times. Results are displayed as the mean \pm standard error of the mean. ${ }^{* *} \mathrm{P}<0.01$ vs. respective controls. MASTL, microtubule associated serine/threonine kinase-like; TNF- $\alpha$, tumor necrosis factor $\alpha$; IL-6, interleukin 6; PDTC, pyrrolidine dithiocarbamate; ChIP, chromatin immunoprecipitation; $\mathrm{IgG}$, immunoglobulin G.

inhibitor NSC74859 were employed to investigate which signaling pathway may be involved in MASTL expression in liver cancer cells, in response to IL-6 and TNF- $\alpha$ stimulation. PDTC, but not NSC74859 influenced MASTL expression, suggesting a role for NF- $\mathrm{KB}$ in this process. The methylation of $\mathrm{H} 3 \mathrm{~K} 4$ and $\mathrm{H} 3 \mathrm{~K} 36$ are associated with transcriptional activation; however, $\mathrm{H} 3 \mathrm{~K} 4 \mathrm{me} 3$ is close to the transcriptional start sites of actively transcribed genes, and as such, is enriched in transcriptionally active promoters $(25,30)$. In the present study it was confirmed that the stimulation of liver cancer cells with IL-6 or TNF- $\alpha$ promoted H3K4Me3 at the MASTL promoter to facilitate chromatin accessibility. Further investigation is required to explain how IL-6 and TNF- $\alpha$ signaling increases the H3K4Me3 level. It was also noted that NF- $\mathrm{KB}$ was involved in the induction of MASTL mRNA expression. Subsequently, future studies intend to investigate the underlying mechanisms 
of $N F-\kappa B$ in this setting, and include the potential role of $\mathrm{NF}-\kappa \mathrm{B}$ in methylation of $\mathrm{H} 3 \mathrm{~K} 4$ at the MASTL promoter.

Previous studies have illustrated that GWL regulates mitosis in Drosophila and Xenopus eggs $(5,34)$. An RNAi assay was employed to investigate the function of MASTL in the liver cancer cell lines HepG2 and SUN387. The results revealed that HepG2 cells were arrested at the $\mathrm{G} 2 / \mathrm{M}$ phase following silencing of $M A S T L$, and that an increase in the number of multinuclear cells was apparent, compared with the control. These results suggested that mitotic regulation of MASTL in human cells was universal.

Uncontrolled proliferation is one of the hallmark characteristics of cancer cells. Normal progression of the cell cycle and subsequent cellular proliferation are gene regulated $(35,36)$, and abnormal expression of such genes as cellular tumor antigen p53 results in uncontrolled cell proliferation (37). The present study revealed that MASTL had a kinase-dependent influence on proliferation in liver cancer cell lines (HepG2 and SUN387). Also, the effects of MASTL on cell proliferation were confirmed by a marked inhibition following gene silencing. On the contrary, cell proliferation was promoted by overexpression of wild-type $M A S T L$, but not the kinase-dead mutant. Further morphological studies revealed that smaller nuclei were observed following the overexpression of MASTL. This indicated that the cells entered mitosis without completing the $\mathrm{S}$ phase, resulting in abnormal cell division.

Though human MASTL has been extensively studied, and numerous studies indicate its upregulation in human oral squamous cell carcinoma (38), little is known of its function in human digestive diseases. Therefore, the role of MASTL in liver cancer was the focus of the present study. IHF analysis revealed higher expression of the MASTL protein in liver cancer tissues compared with those in non-cancerous liver tissues. Moreover, these results indicated that $M A S T L$ expression was induced by TNF- $\alpha$ and IL-6, which was in accordance with the increased concentrations of IL- 6 and TNF- $\alpha$ in the sera of liver cancer patients with chronic HBV infection. These inflammatory cytokines were elevated in chronic hepatitis and liver cancer, supporting their role in the development of the disease.

Taken together, the present study has revealed that the proinflammatory cytokines TNF- $\alpha$ and IL- 6 are elevated in patients with chronic HBV infection. These cytokines induce MASTL expression by increasing H3K4Me3 and activating NF- $\kappa \mathrm{B}$. Abnormal expression of MASTL promotes the proliferation of hepatocytes by regulating mitosis, which subsequently leads to the carcinogenesis of liver cancer.

\section{Acknowledgements}

Not applicable.

\section{Funding}

The present study was supported by the Hebei Province Financial Department Foundation of China (grant no. 361007).

\section{Availability of data and materials}

The datasets used and/or analyzed during the present study are available from the corresponding author on reasonable request.

\section{Authors' contributions}

LC, WL, SC and QZ were responsible for the conceptualization of the study. LC, WL, JY, YW, ZH, YC and JZ curated the data, and LC, WL, DL, HZ and RZ conducted the formal analysis. Investigations were conducted by LC, WL, JY, YW, and $\mathrm{ZH}$, and the methods were designed by LC, WL, DL, YC, HZ, RZ and JZ. Project administration was the responsibility of LC, WL, SC and QZ. Resources were provided by SC and QZ, and the study was supervised by WL, SC and QZ. The original article was written by LC, WL, SC and QZ, and revised by WL, SC and QZ.

\section{Ethics approval and consent to participate}

The present study was approved by the Ethics Committee of The Affiliated Hospital of Hebei University, and written informed consent was obtained from all participants.

\section{Patient consent for publication}

Not applicable.

\section{Competing interests}

The authors declare that they have no competing interests.

\section{References}

1. Nasmyth K: Viewpoint: Putting the cell cycle in order. Science 274: 1643-1645, 1996.

2. Murray AW: Recycling the cell cycle: Cyclins revisited. Cell 116: 221-234, 2004.

3. Davydenko O, Schultz RM and Lampson MA: Increased CDK1 activity determines the timing of kinetochore-microtubule attachments in meiosis I. J Cell Biol 202: 221-229, 2013.

4. Chang HY, Jennings PC, Stewart J, Verrills NM and Jones KT: Essential role of protein phosphatase $2 \mathrm{~A}$ in metaphase II arrest and activation of mouse eggs shown by okadaic acid, dominant negative protein phosphatase 2A, and FTY720. J Biol Chem 286: 14705-14712, 2011.

5. Yu J, Fleming SL, Williams B, Williams EV, Li Z, Somma P, Rieder CL and Goldberg ML: Greatwall kinase: A nuclear protein required for proper chromosome condensation and mitotic progression in Drosophila. J Cell Biol 164: 487-492, 2004.

6. Wang P, Malumbres M and Archambault V: The Greatwall-PP2A axis in cell cycle control. Methods Mol Biol 1170: 99-111, 2014.

7. Cundell MJ, Bastos RN, Zhang T, Holder J, Gruneberg U, Novak B and Barr FA: The BEG (PP2A-B55/ENSA/Greatwall) pathway ensures cytokinesis follows chromosome separation. Mol Cell 52: 393-405, 2013.

8. Voets E and Wolthuis RM: MASTL is the human orthologue of Greatwall kinase that facilitates mitotic entry, anaphase and cytokinesis. Cell Cycle 9: 3591-3601, 2010.

9. Nagel R, Stigter-van Walsum M, Buijze M, van den Berg J, van der Meulen IH, Hodzic J, Piersma SR, Pham TV, Jiménez CR, van Beusechem VW and Brakenhoff RH: Genome-wide siRNA screen identifies the radiosensitizing effect of downregulation of MASTL and FOXM1 in NSCLC. Mol Cancer Ther 14: 1434-1444, 2015.

10. Johnson HJ, Gandhi MJ, Shafizadeh E, Langer NB, Pierce EL, Paw BH, Gilligan DM and Drachman JG: In vivo inactivation of MASTL kinase results in thrombocytopenia. Exp Hematol 37: 901-908, 2009.

11. El-Serag HB and Rudolph KL: Hepatocellular carcinoma: Epidemiology and molecular carcinogenesis. Gastroenterology 132: 2557-2576, 2007.

12. Grivennikov SI, Greten FR and Karin M: Immunity, inflammation, and cancer. Cell 140: 883-899, 2010. 
13. Hoshida Y, Toffanin S, Lachenmayer A, Villanueva A, Minguez B and Llovet JM: Molecular classification and novel targets in hepatocellular carcinoma: Recent advancements. Semin Liver Dis 30: $35-51,2010$.

14. Hartwell LH and Kastan MB: Cell cycle control and cancer. Science 266: 1821-1828, 1994.

15. Chaturvedi P, Eng WK, Zhu Y, Mattern MR, Mishra R, Hurle MR, Zhang X, Annan RS, Lu Q, Faucette LF, et al: Mammalian Chk2 is a downstream effector of the ATM-dependent DNA damage checkpoint pathway. Oncogene 18: 4047-4054, 1999.

16. Zeng JZ, Wang HY, Chen ZJ, Ullrich A and Wu MC: Molecular cloning and characterization of a novel gene which is highly expressed in hepatocellular carcinoma. Oncogene 21: 4932-4943, 2002.

17. Bishayee A: The role of inflammation and liver cancer. Adv Exp Med Biol 816: 401-435, 2014

18. Hodge DR, Hurt EM and Farrar WL: The role of IL-6 and STAT3 in inflammation and cancer. Eur J Cancer 41: 2502-2512, 2005.

19. Pfaffi MW: A new mathematical model for relative quantification in real-time RT-PCR. Nucleic Acids Res 29: e45, 2001.

20. El-Serag HB: Hepatocellular carcinoma. N Engl J Med 365: 1118-1127, 2011.

21. Naugler WE, Sakurai T, Kim S, Maeda S, Kim K, Elsharkawy AM and Karin M: Gender disparity in liver cancer due to sex differences in MyD88-dependent IL-6 production. Science 317: 121-124, 2007.

22. Park EJ, Lee JH, Yu GY, He G, Ali SR, Holzer RG, Osterreicher $\mathrm{CH}$, Takahashi $\mathrm{H}$ and Karin $\mathrm{M}$ : Dietary and genetic obesity promote liver inflammation and tumorigenesis by enhancing IL-6 and TNF expression. Cell 140: 197-208, 2010

23. He G, Dhar D, Nakagawa H, Font-Burgada J, Ogata H, Jiang Y, Shalapour S, Seki E, Yost SE, Jepsen K, et al: Identification of liver cancer progenitors whose malignant progression depends on autocrine IL-6 signaling. Cell 155: 384-396, 2013.

24. Iliopoulos D, Hirsch HA and Struhl K: An epigenetic switch involving NF-kappaB, Lin28, Let-7 MicroRNA, and IL6 links inflammation to cell transformation. Cell 139: 693-706, 2009.

25. Pilati C, Amessou M, Bihl MP, Balabaud C, Nhieu JT, Paradis V, Nault JC, Izard T, Bioulac-Sage P, Couchy G, et al: Somatic mutations activating STAT3 in human inflammatory hepatocellular adenomas. J Exp Med 208: 1359-1366, 2011.

26. Mochida S, Maslen SL, Skehel M and Hunt T: Greatwall phosphorylates an inhibitor of protein phosphatase 2A that is essential for mitosis. Science 330: 1670-1673, 2010.
27. Gharbi-Ayachi A, Labbé JC, Burgess A, Vigneron S, Strub JM, Brioudes E, Van-Dorsselaer A, Castro A and Lorca T: The substrate of Greatwall kinase, Arpp19, controls mitosis by inhibiting protein phosphatase 2A. Science 330: 1673-1677, 2010.

28. Burgess A, Vigneron S, Brioudes E, Labbé JC, Lorca T and Castro A: Loss of human Greatwall results in G2 arrest and multiple mitotic defects due to deregulation of the cyclin B-Cdc2/PP2A balance. Proc Natl Acad Sci USA 107: 12564-12569, 2010.

29. Ghosh S and Karin M: Missing pieces in the NF-kappaB puzzle. Cell 109 (Suppl): S81-S96, 2002.

30. Xing S, Zhang B, Hua R, Tai WC, Zeng Z, Xie B, Huang C, Xue J, Xiong S, Yang J, et al: URG4/URGCP enhances the angiogenic capacity of human hepatocellular carcinoma cells in vitro via activation of the NF- $\kappa$ B signaling pathway. BMC Cancer 15: 368, 2015.

31. Wang Y, Tu Q, Yan W, Xiao D, Zeng Z, Ouyang Y, Huang L, Cai J, Zeng X, Chen YJ and Liu A: CXC195 suppresses proliferation and inflammatory response in LPS-induced human hepatocellular carcinoma cells via regulating TLR4-MyD88-TAK1-mediated NF- $\kappa$ B and MAPK pathway. Biochem Biophys Res Commun 456: 373-379, 2015.

32. Lu X, Ma P, Shi Y, Yao M, Hou L, Zhang P and Jiang L: NF-кB increased expression of 17 $\beta$-hydroxysteroid dehydrogenase 4 promotes HepG2 proliferation via inactivating estradiol. Mol Cell Endocrinol 401: 1-11, 2015.

33. He G and Karin M: NF- $\mathrm{BB}$ and STAT3-key players in liver inflammation and cancer. Cell Res 21: 159-168, 2011.

34. Yu J, Zhao Y, Li Z, Galas S and Goldberg ML: Greatwall kinase participates in the $\mathrm{Cdc} 2$ autoregulatory loop in Xenopus egg extracts. Mol Cell 22: 83-91, 2006.

35. Qian Y and Chen X: Tumor suppression by p53: Making cells senescent. Histol Histopathol 25: 515-526, 2010.

36. Hanahan D and Weinberg RA: The hallmarks of cancer. Cell 100: 57-70, 2000.

37. Hermeking H: MicroRNAs in the 53 network: Micromanagement of tumour suppression. Nat Rev Cancer 12: 613-626, 2012.

38. Lorca $\mathrm{T}$ and Castro A: The Greatwall kinase: A new pathway in the control of the cell cycle. Oncogene 32: 537-543, 2013.

This work is licensed under a Creative Commons

Attribution-NonCommercial-NoDerivatives 4.0 International (CC BY-NC-ND 4.0) License. 